\title{
Is Knee Joint Distraction a Viable Treatment Option for Knee OA?-A Literature Review and Meta-Analysis
}

\author{
Tsuneari Takahashi, MD, PhD ${ }^{1,2}$ Thomas G. Baboolal, $\mathrm{PhD}^{1}$ Jonathan Lamb, MRCS ${ }^{1,3}$ \\ Thomas W. Hamilton, MRCS ${ }^{4, *}$ Hemant G. Pandit, FRCS, DPhil ${ }^{1,3,4}$ \\ ${ }^{1}$ Leeds Institute of Rheumatic and Musculoskeletal Medicine, \\ University of Leeds, Leeds, United Kingdom \\ ${ }^{2}$ Department of Orthopedic Surgery, Jichi Medical University, \\ Shimotsuke, Japan \\ ${ }^{3}$ Department of Orthopedic Surgery, Chapel Allerton Hospital, \\ Leeds Teaching Hospital NHS Trust, Leeds, United Kingdom \\ ${ }^{4}$ Nuffield Department of Orthopaedics, Rheumatology and \\ Musculoskeletal Sciences, University of Oxford, Oxford, United Kingdom

\begin{abstract}
Address for correspondence Tsuneari Takahashi, MD, PhD, 2nd Floor, Chapel Allerton Hospital, Chapeltown Road, Leeds LS7 4SA, United Kingdom (e-mail: tsuneari9@jichi.ac.jp).
\end{abstract} \\ J Knee Surg 2019;32:788-795.
}

\begin{abstract}
Knee joint distraction (KJD) is a new application of an established technique to regenerate native cartilage using an external fixator. The purpose of this study is to perform a systematic review and meta-analysis of the literature to determine whether KJD is beneficial for knee osteoarthritis and how results compare with established treatments. Studies assessing the outcomes of KJD were retrieved, with three studies (one cohort and two randomized controlled trials), 62 knees, meeting the inclusion criteria. The primary outcome was functional outcome, assessed using a validated outcome score, at 1 year. Secondary outcomes included pain scores, structural assessment of the joint, and adverse events. KJD is associated with improvements in Western Ontario and McMaster Universities Osteoarthritis Index (WOMAC) from baseline to 1 year as well as reductions in pain scores and improvements in structural

Keywords

- knee osteoarthritis

- knee joint distraction

- total knee arthroplasty

- high tibial osteotomy

- outcomes

- complication parameters assessed radiographically and by magnetic resonance imaging. KJD is not associated with decreased knee flexion, but is associated with a high risk of pin site infection. In patients aged 65 years or under at 1 year, no differences in WOMAC or pain scores was detected between patients managed with KJD compared with high tibial osteotomy or total knee arthroplasty. KJD may represent a potential treatment for knee arthritis, though further trials with longer term follow-up are required to establish its efficacy compared with contemporary treatments. This is a Level I (systematic review and meta-analysis) study.
\end{abstract}

Knee osteoarthritis $(\mathrm{OA})$ is the most common musculoskeletal disease estimated to affect $3.8 \%$ of the world's population. ${ }^{1}$ Considered a disease of the whole joint, knee OA is characterized by loss of cartilage, bone remodeling, and inflammation. Cumulative joint degeneration eventually

Thomas W. Hamilton is the senior co-author of the study. leads to substantial loss of function and quality of life, and represents a major cause of global disability. ${ }^{1,2}$ The burden of $\mathrm{OA}$ is set to increase with rising obesity levels and an aging population. ${ }^{1,3}$ Gold standard treatment for OA of significant severity is joint arthroplasty after initial conservative treatment. Beyond arthroplasty, no other treatment is proven effective in halting or reversing disease

Copyright @ 2019 by Thieme Medical Publishers, Inc., 333 Seventh Avenue, New York, NY 10001, USA. Tel: +1(212) 584-4662.
DOI https://doi.org/ 10.1055/s-0038-1669447. ISSN 1538-8506. 
progression. Globally, the prevalence of knee OA peaks at 50 years. ${ }^{1}$ However, both patients and surgeons are reluctant to replace joints where the patient is expected to outlive the lifespan of the prosthesis as there is a greater risk of revision surgery. ${ }^{4-6}$

Consequently, there is an increasing need for alternative treatments for this younger OA population, not least because of the increased failure risk ${ }^{5}$ but also because in some cases arthroplasty may result in poor clinical outcomes. ${ }^{7}$ Following injury and osteoarthritis in the ankle, ankle joint distraction has provided a useful means of reducing pain, improving function, and increasing radiological joint space. ${ }^{8}$ Likewise, basilar thumb arthritis has been effectively treated with joint distraction and debridement in small prospective studies. ${ }^{9}$ There are a certain risks of infection at pin sites and related bone infection often observed in any surgical procedure using external frame and pins or wires; however, such joint sparing alternatives are useful for patients who wish to preserve the native joint.

A similar approach has been adopted to treat knee OA with knee joint distraction (KJD). KJD uses an external fixator to unload the joint by distracting the tibia and femur. ${ }^{10}$ It is reported that this temporary mechanical unloading allows natural intrinsic repair processes to regenerate cartilaginous tissue evidenced by a sustained clinical benefit and increase in joint width space. ${ }^{11}$ With KJD being a joint sparing procedure aimed at postponing a first prosthesis, successful clinical adoption could significantly improve patients' quality of life and thus reduce the long-term health care costs associated with knee OA.

The aims of this systematic review are to identify and examine the current evidence for the use of KJD focusing on clinical and radiological outcomes. This review will also help to identify gaps in our understanding and so inform future clinical and scientific studies.

\section{Materials and Methods}

\section{Inclusion and Exclusion Criteria}

Eligible studies included those involving patients aged 18 years or older with knee arthritis that compared surgical KJD against other surgical procedures for knee arthritis. There were no exclusion-based study designs or duration of distraction.

\section{Information Sources and Search Strategy}

Electronic databases (MEDLINE [Ovid], EMBASE [Ovid], Web of Science [ISI Web of Knowledge]) were searched from their inception until 25 February 2018 for studies meeting the inclusion criteria. Searches were tailored to individual databases with the search strategy for MEDLINE shown in - Appendix A. In addition, reference lists of reviews and retrieved articles were assessed for further studies as were registers of controlled clinical trials (metaRegister of controlled trials [mRCT] [www.controlled-trials.com/mrct], clinicaltrials.gov [www.clinicaltrials.gov] and the World Health Organization [WHO] International Clinical Trials Registry Platform [ICTRP] [http://apps.who.int/trialsearch/]). No restrictions were applied based on the publication status. Where necessary authors were contacted for additional information.

Studies were assessed independently in duplicate for eligibility and data from eligible studies extracted independently in duplicate into an electronic database (T.T., T.W.H.). A risk of bias assessment was performed on included studies.

\section{Outcome Measures Assessed}

To assess the outcome of KJD, improvements from baseline to 1 year post intervention were assessed. To compare KJD with other surgical interventions, outcomes at 1 year post intervention were assessed.

The primary outcome assessed was functional outcome, assessed using a validated outcome score, at 1 year following surgical intervention. Secondary outcomes included pain scores, assessed using a validated pain score, structural assessment of the joint, both radiographic and with magnetic resonance imaging (MRI), and assessment of adverse events. All secondary outcomes were assessed at 1 year following surgical intervention.

\section{Statistical Analysis}

Heterogeneity of included studies was assessed using the $\mathrm{I}^{2}$ statistic and in the event of substantial heterogeneity $\left(\mathrm{I}^{2}>85 \%\right)$, a meta-analysis was not be performed. As a degree of variability was expected due to the subjectivity of the outcome measures, a random-effects model was used in all cases. For continuous data, the mean difference (MD) was calculated along with $95 \%$ confidence intervals (95\% $\mathrm{CI}$ ), calculated using the inverse variance method. For dichotomous data, the risk difference along with 95\% CI was calculated using the Cochran-Mantel-Haenszel method. Data analysis was performed using standard statistical techniques as described in the Cochrane Handbook for Systematic Reviews of Interventions, using Review Manager-5.3 (The Nordic Cochrane Centre, The Cochrane Collaboration, 2014).

\section{Results}

Three studies consisting of one cohort study and two randomized controlled trials were identified as meeting inclusion criteria $^{11-13}$ (-Fig. 1). The results of the cohort study were reported across three papers with relevant data extracted where reported. ${ }^{11,14,15}$ Included studies are outlined in -Table 1 with an assessment of risk of bias presented in -Fig. 2. All studies were considered at high risk of performance and detection bias as it was not possible to blind surgeons, participants, or outcome assessors as to the treatment received. Attrition and reporting bias were assessed as low risk with no loss to follow-up at 1 year reported. As all three studies originate from the same research group, it was considered that this presented an unclear risk of bias.

Two studies were excluded as they reported the results of arthroscopic microfracture in combination with KJD and it was the authors opinion that, as microfracture is already an established treatment for cartilaginous loss, it would not be possible to delineate any treatment effect seen. ${ }^{16,17}$ The first 


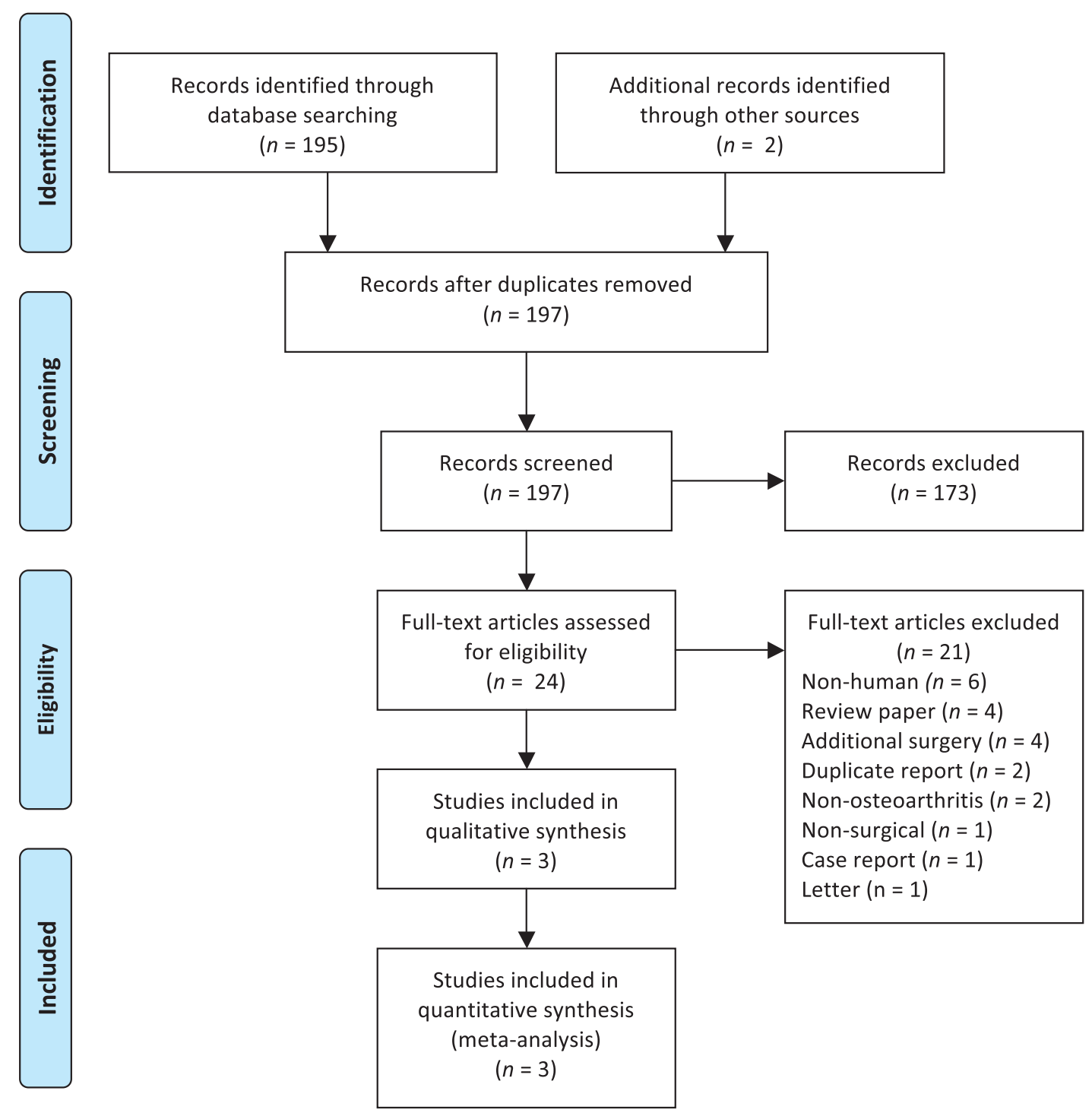

Fig. 1 PRISMA flow diagram.

of these studies by Deie et al reported the outcomes of six knees managed with KJD and microfracture and found at a mean 3-year follow-up significant improvements in Japanese Orthopaedic Association Score, VAS pain score, and radiographic joint space width. ${ }^{16}$ The second, by Aly et al, reported the outcomes of 61 knees, 19 managed with KJD, joint debridement and microfracture and 42 managed with joint debridement and microfracture and found that at a mean follow-up of 3 to 5 years the group managed with KJD, joint debridement, and microfracture had significantly improved pain, walking capacity, stair climbing, and radio- graphic joint space width compared with baseline, whereas those treated with joint debridement and microfracture without KJD did not. ${ }^{17}$

\section{Outcomes of KJD Improvement from Baseline to One Year Post Intervention}

\section{Primary Outcome}

The Western Ontario and McMaster Universities Osteoarthritis Index (WOMAC) scores at baseline and 1 year post KJD were reported in all three studies, 62 patients, with a

Table 1 List of included studies

\begin{tabular}{|l|l|l|l|l|}
\hline Author & Procedure & Age & Male gender & BMI \\
\hline van der Woude et al $2017^{11}$ & KJD vs. OA & 48.5 vs. 51.2 & $55 \%$ vs. $45 \%$ & 29.6 vs. 31.1 \\
\hline van der Woude et al $2017^{12}$ & KJD vs. TKA & 54.9 vs. 56.2 & $45 \%$ vs. $36 \%$ & 27.4 vs. 29.4 \\
\hline van der Woude et al $2017^{13}$ & KJD vs. HTO & 51.2 vs. 49.4 & $73 \%$ vs. $60 \%$ & 27.5 vs. 27.2 \\
\hline
\end{tabular}

Abbreviations: BMI, body mass index; HTO, high tibial osteotomy; KJD, knee joint distraction; OA, osteoarthritis; TKA, total knee arthroplasty. 




Fig. 2 Risk of bias summary.

significant improvement in WOMAC scores, MD 28.7 points ( $p<0.001 ; 95 \% \mathrm{CI}, 22.6-34.8$ ), between baseline and 1 year post surgery observed (-Fig. 3 ). Improvements were seen across all subdomains of WOMAC: pain $(p \leq 0.001$; MD 29.3 points $95 \% \mathrm{CI}, 21.9-36.5)$, stiffness ( $p \leq 0.001$; MD 19.5 points $95 \% \mathrm{CI}, 8.4-30.6$ ), and function ( $p \leq 0.001$; MD 29.5 points $95 \% \mathrm{CI}, 23.6-35.4)$.

Knee injury and Osteoarthritis Outcome Score (KOOS), Intermittent and Constant Osteoarthritis Pain (ICOAP) score, EuroQol 5 Dimensions (EQ-5D), and Short form (SF)-36 were reported in two studies, 42 patients. Significant improvements between baseline and 1 year scores were observed for KOOS ( $p<0.001$, MD 23.2 points 95\% CI, 15.4-31.1), ICOAP $(p<0.001$, MD 26.7 points 95\% CI, 17.0-36.4), and EQ-5D $(p<0.001$, MD 0.15 points $95 \% \mathrm{CI}, 0.06-0.23)$ and all subdomains. Significant improvements between baseline and 1 year SF-36 physical component score $(p=0.009$, MD
7.8 points $95 \% \mathrm{CI}, 1.9-13.7)$, but not mental component score ( $p=0.41, \mathrm{MD}-1.5$ points $95 \% \mathrm{Cl},-5.0-2.0$ ) were observed.

\section{Secondary Outcomes}

Pain score, assessed using a pain visual analog score (VAS) 0 to 100 where 0 was equivalent to no pain, was reported in all three studies, 62 patients. Patients managed with KJD reported significant improvements in pain VAS of 33.3 points ( $p \leq 0.001 ; 95 \% \mathrm{CI}, 19.7-46.9$ ) from baseline to 1 year post surgery (-Fig. 4 ).

Structural assessment of the joint was performed radiographically in all three studies, 59 patients, and by MRI in one study, 20 patients. Between baseline and 1 year following $\mathrm{KJD}$, the radiographic minimum joint space width increased by $0.8 \mathrm{~mm}(p<0.001 ; 95 \% \mathrm{CI}, 0.5-1.0 ;$ - Fig. 5$)$ and mean joint space width increased by $0.8 \mathrm{~mm}(p=0.003 ; 95 \% \mathrm{CI}$, $0.3-1.3$ ). On MRI, the mean cartilage thickness over the total

\begin{tabular}{|c|c|c|c|c|c|c|c|c|c|}
\hline \multirow[b]{2}{*}{ Study or Subgroup } & \multicolumn{3}{|c|}{1 year } & \multicolumn{3}{|c|}{ Baseline } & \multirow{2}{*}{\multicolumn{2}{|c|}{$\begin{array}{ll} & \text { Mean Difference } \\
\text { Weight } & \mathrm{N}, \text { Random, } 95 \% \mathrm{Cl} \\
\end{array}$}} & \multirow{2}{*}{$\begin{array}{c}\text { Mean Difference } \\
\text { IV, Random, } 95 \% \mathrm{Cl}\end{array}$} \\
\hline & Mean & SD & Total & Mean & SD & Total & & & \\
\hline van der Woude $2017^{1}$ & 76.3 & 21.4663 & 20 & 43.9 & 14.758 & 20 & $27.9 \%$ & $32.40(20.98,43.82)$ & \multirow{3}{*}{$\longrightarrow$} \\
\hline van der Woude $2017^{2}$ & 76 & 17 & 22 & 54 & 20 & 22 & $30.2 \%$ & $22.00(11.03,32.97)$ & \\
\hline van der Woude $2017^{3}$ & 80 & 18 & 20 & 49 & 11 & 20 & $41.9 \%$ & $31.00(21.75,40.25)$ & \\
\hline Total $(95 \% \mathrm{Cl})$ & & & 62 & & & 62 & $100.0 \%$ & $28.68(22.55,34.80)$ & \\
\hline \multicolumn{9}{|c|}{$\begin{array}{l}\text { Heterogeneity: } \mathrm{Tau}^{2}=1.07 ; \mathrm{Chi}^{2}=2.07, \mathrm{df}=2(p=0.35) ; \mathrm{I}^{2}=4 \% \\
\text { Test for overall effect: } Z=9.17(p<0.00001)\end{array}$} & Improvement \\
\hline
\end{tabular}

Fig. 3 Forest plot of improvement from baseline to 1-year Western Ontario and McMaster Universities Osteoarthritis Index scores in knees managed with knee joint distraction. $\mathrm{Cl}$, confidence interval; SD, standard deviation. 
792 Is KJD a Viable Treatment Option for Knee OA? Takahashi et al.

\begin{tabular}{|c|c|c|c|c|c|c|c|c|c|}
\hline \multirow[b]{2}{*}{ Study or Subgroup } & \multicolumn{3}{|c|}{ Baseline } & \multicolumn{3}{|c|}{1 year } & \multicolumn{2}{|r|}{ Mean Difference } & \multirow{2}{*}{$\begin{array}{c}\text { Mean Difference } \\
\text { I, Random, } 95 \% \mathrm{Cl}\end{array}$} \\
\hline & Mean & SD & Total & Mean & SD & Total & Weight & IV, Random, $95 \% \mathrm{Cl}$ & \\
\hline van der Woude $2017^{1}$ & 72.9 & 9.3915 & 20 & 30.5 & 25.9384 & 20 & $35.9 \%$ & $42.40(30.31,54.49)$ & $\longrightarrow$ \\
\hline van der Woude $2017^{2}$ & 55 & 24 & 22 & 36 & 26 & 22 & $31.5 \%$ & $19.00(4.21,33.79)$ & \\
\hline van der Woude $2017^{3}$ & 65 & 19 & 20 & 28 & 26 & 20 & $32.6 \%$ & $37.00(22.89,51.11)$ & \\
\hline Total $(95 \% \mathrm{Cl})$ & & & 62 & & & 62 & $100.0 \%$ & $33.27(19.67,46.87)$ & \\
\hline \multicolumn{9}{|c|}{$\begin{array}{l}\text { Heterogeneity: } \text { Tau }^{2}=96.05 ; \mathrm{Chi}^{2}=5.98, \mathrm{df}=2(p=0.05) ; \mathrm{I}^{2}=67 \% \\
\text { Test for overall effect: } Z=4.79(p<0.00001)\end{array}$} & $\begin{array}{cc}1 & 15 \\
\text { Improvement }\end{array}$ \\
\hline
\end{tabular}

Fig. 4 Forest plot of improvement from baseline to 1-year visual analog scores for pain (0-100) in knees managed with knee joint distraction. $\mathrm{Cl}$, confidence interval; SD, standard deviation.

\begin{tabular}{|c|c|c|c|c|c|c|c|c|c|}
\hline \multirow[b]{2}{*}{ Study or Subgroup } & \multicolumn{2}{|r|}{1 year } & \multicolumn{4}{|c|}{ Baseline } & \multicolumn{2}{|r|}{ Mean Difference } & \multirow{2}{*}{$\begin{array}{c}\text { Mean Difference } \\
\text { N, Random, } 95 \% \mathrm{Cl}\end{array}$} \\
\hline & Mean & SD & Total & Mean & SD & Total & Weight & IV, Random, $95 \% \mathrm{Cl}$ & \\
\hline van der Woude $2017^{1}$ & 1.7 & 1.3416 & 20 & 1.2 & 1.3416 & 20 & $9.0 \%$ & $0.50(-0.33,1.33)$ & \\
\hline van der Woude $2017^{2}$ & 1.4 & 0.469 & 22 & 0.6 & 0.469 & 22 & $80.7 \%$ & $0.80(0.52,1.08)$ & \\
\hline van der Woude $2017^{3}$ & 1.5 & 1.1 & 17 & 0.6 & 1.2 & 17 & $10.4 \%$ & $0.90(0.13,1.67)$ & \\
\hline Total $(95 \% \mathrm{CI})$ & & & 59 & & & 59 & $100.0 \%$ & $0.78(0.53,1.03)$ & \\
\hline \multicolumn{9}{|c|}{$\begin{array}{l}\text { Heterogeneity: } \mathrm{Tau}^{2}=0.00 ; \mathrm{Chi}^{2}=0.55, \mathrm{df}=2(p=0.76) ; \mathrm{I}^{2}=0 \% \\
\text { Test for overall effect: } \mathrm{Z}=6.17(p<0.00001)\end{array}$} & $\begin{array}{lc}0 & 1 \\
\text { Improvement }\end{array}$ \\
\hline
\end{tabular}

Fig. 5 Forest plot of improvement in radiographic minimum joint space width $(\mathrm{mm})$ in the affected tibiofemoral compartment from baseline to one year in knees managed with knee joint distraction. $\mathrm{Cl}$, confidence interval; SD, standard deviation.

subchondral bone area increased from 1.4 (standard deviation, SD 0.3 ) to $1.6 \mathrm{~mm}(\mathrm{SD} 0.3 ; p=0.03$ ) on the tibia and from 1.0 (SD 0.4) to $1.4 \mathrm{~mm}$ (SD $0.3 ; p<0.001$ ) on the femur. The percentage of denuded subchondral bone decreased from 16.7 (SD 17.2) to $4.8 \%$ (SD 8.3; $p=0.006$ ) on the tibia and from 27.3 (SD 25.6) to $4.2 \%$ (SD 10.2; $p<0.001$ ) on the femur.

\section{Adverse Events}

Knee flexion was reported in two studies, 42 patients. No change in knee flexion between baseline and 1 year following KJD was observed ( $p=0.18$; MD $2.4^{\circ} 95 \% \mathrm{CI},-1.1-5.9$ ) from baseline to 1 year post surgery. Across all three studies, 62 patients, one patient was reported as requiring manipulation under anesthetic (MUA) at 17 days following frame removal for stiffness.

Across all three studies, 62 patients, 42 patients developed single or multiple pin site infection requiring antibio- tics. Overall, the risk of developing pin site infection was $69 \%$ (95\% CI, 51-87) (-Fig. 6). The risk of developing pin site infection requiring oral antibiotics was $57 \%$ (95\% CI, 33-82). The risk of developing pin site infection requiring intravenous antibiotics was $10 \%(95 \% \mathrm{CI}, 1-18)$. Overall two patients required surgical irrigation and debridement with one developing osteomyelitis 3 weeks following frame removal.

Additional adverse events reported with the use of KJD included pulmonary emboli ( 2 of 20 patients [10\%] in one study), postoperative foot drop managed with ankle foot orthosis ( 1 patient), failure of the KJD device ( 1 patient), and breaking of a bone pin during application ( 1 patient).

\section{Outcomes of KJD Compared with Other Treatments}

Primary Outcome

Two randomized controlled trials assessed the outcomes of KJD against other treatments for arthritis, one against high

\begin{tabular}{|c|c|c|c|c|c|}
\hline Study or Subgroup & Events & Total & Weight & $\begin{array}{c}\text { Risk Difference } \\
\text { M-H, Random, } 95 \% \mathrm{Cl}\end{array}$ & $\begin{array}{c}\text { Risk Difference } \\
\text { M-H, Random, } 95 \% \mathrm{Cl}\end{array}$ \\
\hline van der Woude $2017^{1}$ & 17 & 20 & $37.2 \%$ & $0.85(0.68,1.02)$ & $\longrightarrow$ \\
\hline van der Woude $2017^{2}$ & 13 & 22 & $32.0 \%$ & $0.59(0.38,0.80)$ & \\
\hline van der Woude $2017^{3}$ & 12 & 20 & $30.8 \%$ & $0.60(0.38,0.82)$ & \\
\hline Total $(95 \% \mathrm{Cl})$ & & 62 & $100.0 \%$ & $0.69(0.51,0.87)$ & \\
\hline Total events & 42 & & & & \\
\hline \multicolumn{5}{|c|}{$\begin{array}{l}\text { Heterogeneity: } \text { Tau }^{2}=0.02 ; \mathrm{Chi}^{2}=4.99, \mathrm{df}=2(p=0.08) ; I^{2}=60 \% \\
\text { Test for overall effect: } Z=7.42(p<0.00001)\end{array}$} & $\begin{array}{c}0.5 \\
\text { Risk Difference }\end{array}$ \\
\hline
\end{tabular}

Fig. 6 Forest plot of risk of pin site infection in knees managed with knee joint distraction. $\mathrm{Cl}$, confidence interval. 


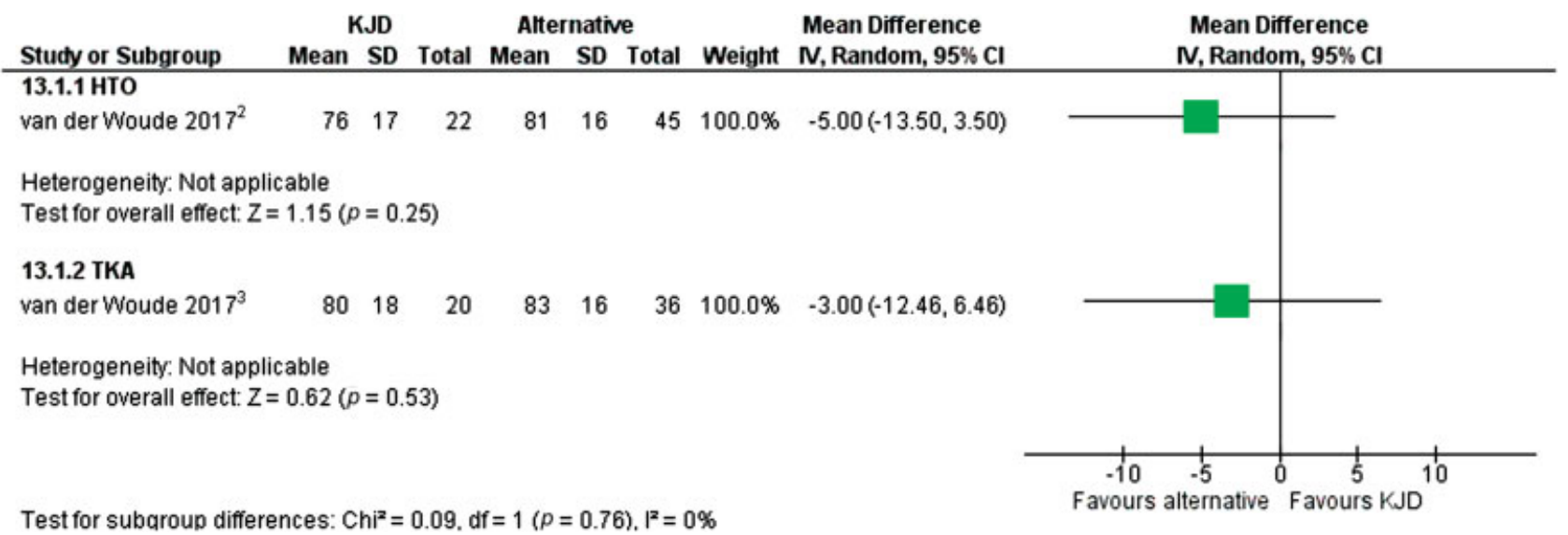

Fig. 7 Forest plot of 1-year Western Ontario and McMaster Universities Osteoarthritis Index scores in knees managed with knee joint distraction (KJD) compared with knees managed with high tibial osteotomy (HTO) and total knee arthroplasty (TKA). Cl, confidence interval; SD, standard deviation.

tibial osteotomy (HTO) and one against total knee arthroplasty (TKA). Both studies were conducted in patients aged 65 years and under. At 1 year, no difference in total WOMAC score, or across subdomains, was seen between knees managed with KJD and those managed with HTO ( $p=0.25$; MD -5.0 points, $95 \% \mathrm{CI},-13.5-3.5)$ or TKA ( $p=0.53$; MD -3.0 points, 95\% CI, -12.5-6.5) (-Fig. 7). At 1 year, no difference was seen in KOOS, ICOAP, EQ-5D or SF-36 between treatment groups.

Pain score, assessed using a pain VAS 0 to 100 , was reported in both studies. At 1 year, no difference in pain VAS was seen between knees managed with KJD and those managed with HTO ( $p=0.17$; MD 9.0 points, $95 \% \mathrm{CI},-3.8-$ 21.8 ) or TKA ( $p=0.13$; MD 10.0 points, $95 \% \mathrm{CI},-3.0-23.0$ ) (-Fig. 8).

\section{Adverse Events}

At 1 year, no difference in knee flexion was seen between knees managed with KJD and those managed with HTO $(p=0.05 ;$ MD 4.0 degrees, $95 \% \mathrm{CI},-0.1-8.1)$ or TKA $(p=0.07 ;$ MD 5.0 degrees, $95 \% \mathrm{CI},-0.3-10.3)$. No difference in the rate of MUA was seen between KJD and HTO ( $p=0.40$; risk difference (RD) $0.0595 \% \mathrm{CI},-0.1-0.2$ ). A higher rate of
MUA was seen with TKA compared with KJD ( $p=0.04$; RD 0.14 95\% CI, 0-0.3).

The risk of developing infection requiring antibiotics was significantly higher following KJD compared with both HTO $(p<0.01$; RD $0.595 \%$ CI, $0.3-0.8)$ and TKA $(p<0.01$; RD 0.6 $95 \% \mathrm{CI}, 0.4-0.8)$. This is likely to be secondary to associated risks of using pins which provide a communication between the external environment and lower limb bones into which they are placed.

\section{Discussion}

The main findings of this systematic review are that KJD is associated with significant improvements in functional scores, pain scores, and radiographic measures of cartilage thickness at 1 year postoperatively, and in patients aged 65 years or younger have comparable functional outcomes to HTO and TKA. The main limitation of KJD is the occurrence of pin-tract infection that was reported in 69\% (95\% CI, 5187 ) of patients and was significantly higher than that seen in HTO or TKA. At 1 year, no difference in knee flexion, compared with baseline flexion and flexion 1 year following HTO and TKA, was seen. While MUA following KJD has been

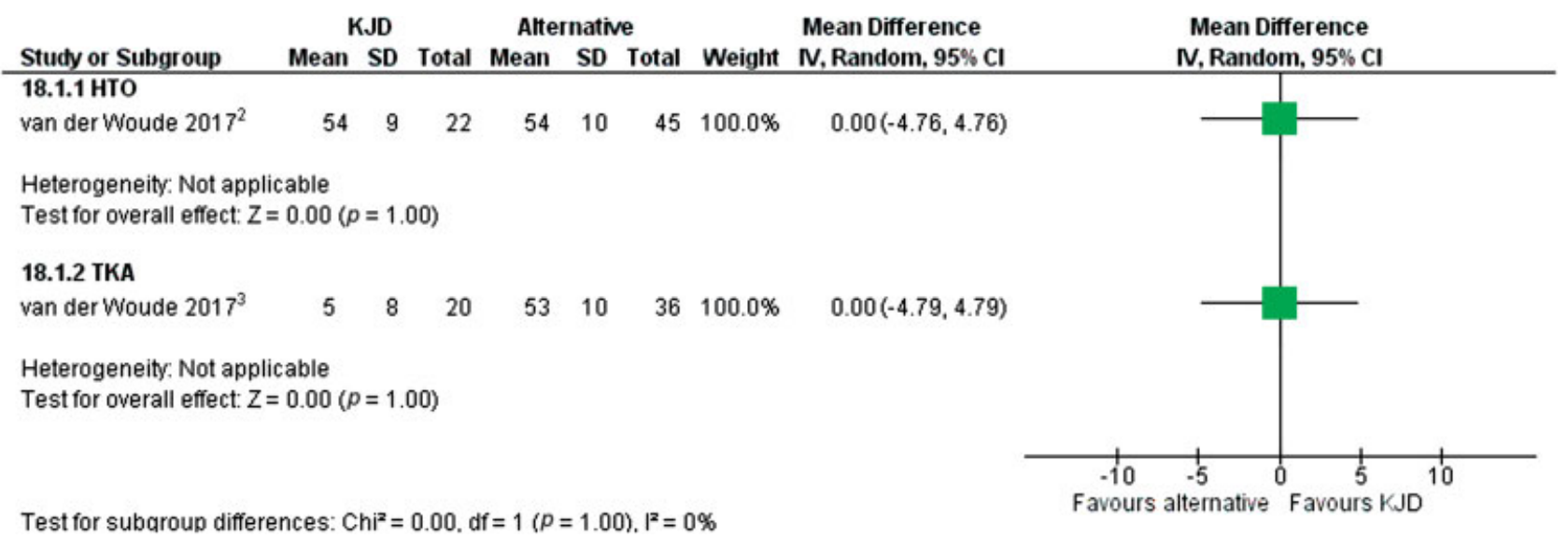

Fig. 8 Forest plot of 1-year visual analog scores for pain (0-100) in knees managed with knee joint distraction (KJD) compared with knees managed with high tibial osteotomy (HTO) and total knee arthroplasty (TKA). Cl, confidence interval; SD, standard deviation. 
reported (one case across three studies, 62 patients), the rate of MUA was found to be significantly lower than the rate observed following TKA.

Compared with older patients, in young patients managed with arthroplasty, the risk of implant failure and subsequent revision burden is high and any intervention that can postpone or reduce the need for the index procedure in this group, and other groups at risk of poor outcomes, is worth considering. This review has found that KJD appears to be a potential alternative treatment option in managing knee OA, and in patients aged 65 years or younger, the results appear to be as good as HTO and TKA at 1 year. While these results are promising, the high rate of pin site infection following $\mathrm{KJD}$ is a concern because both HTO and TKA can give lower rate of postoperative infection. Despite in the majority of these cases, resolution of infection was achieved with oral antibiotics. In a very few instances, osteomyelitis has been reported, and surgeons may well have concerns about performing arthroplasty in these cases should KJD fail. However, Wiegant et al ${ }^{18}$ described the safety to perform TKA following KJD and concluded that it appears safe to treat patients several years following KJD with a TKA.

The mechanism by which KJD works is unclear. In the clinical studies of KJD, increased radiographic JSW and coverage of denuded bone assessed by MRI were reported. Biomarker analysis has reported that following KJD a decrease in the collagen type II breakdown marker is observed coupled with an increase in the collagen type II synthesis marker. ${ }^{14,15}$ While these findings would suggest that KJD changes the intra-articular environment to one that favors cartilage repair. It is likely that the conflicting results obtained in animal experiments are due to a variety of reasons such as differences in experimental setup, type of surrogate endpoints used to assess cartilage repair, and limited follow-up. Some studies have shown promising results with evidence of bone and cartilage repair, while others have failed to demonstrate any advantage with KJD, with some even reporting adverse effect on the cartilage integrity. It is clear from these conflicting observations that more work is needed to establish indeed when and how joint distraction works and in which scenarios. ${ }^{19-26}$

Alongside the mechanism of action of KJD, there are several other areas of uncertainty around this treatment. In the present studies, static distraction was applied using two $45 \mathrm{~kg}$ springs to permit some degree of joint loading. Whether this represent the optimum distraction force, and whether a hinged distractor, which has been demonstrated to be superior for ankle OA, still needs to be assessed. ${ }^{27,28}$ Additionally, the patient population most likely to benefit from distraction and optimum duration of distraction remains to be defined. Early reports suggest that men with more severe arthritis are most likely to respond to treatment, and 6 weeks distraction provides equivalent clinical outcomes to 8 weeks distraction; however, these findings are based on limited data, and appropriately powered trials comparing the outcomes of KJD to other treatments for knee OA are required. ${ }^{29,30}$ Finally, further information on the long-term efficacy of KJD is required. Current data suggest that at 5 years the functional outcomes and structural assessments of joint remain improved compared with baseline, approximately $70 \%$ of the patients treated still have their own knee instead of the initially planned joint prosthesis. ${ }^{11}$ At 9 years post distraction, still $50 \%$ of the patients continue to manage with their own knee and thereby the need for an artificial joint is avoided. Remarkably, mostly women seem to drop out and opt for further intervention, although there is no clear explanation for this gender difference. $^{31}$

The strength of this systematic review is that it is a comprehensive assessment of the efficacy of KJD for the treatment of knee arthritis. The weakness of this review is that it is limited by the data available, with only three studies available for inclusion, with all originating from the same research group.

This study has highlighted that KJD may be a valid alternative to HTO and TKA in the treatment of knee arthritis in the young, resulting in improvements in functional and pain as well as evidence of structural improvements within the joint lasting beyond 1 year. However, further work is required to optimize the technique of $\mathrm{KJD}$, define the optimum population for its use as well as develop methods to reduce the risk of pin site infection, the major complication associated with this technique. Ultimately KJD needs to be assessed pragmatically through appropriately powered multicenter studies designed to assess its long-term effectiveness and comparative efficacy against other established treatments for knee OA.

\section{Ethical Approval}

This is a systematic review so ethical approval was waived.

\section{Funding}

None.

Conflict of interest

None declared.

\section{References}

1 Cross M, Smith E, Hoy D, et al. The global burden of hip and knee osteoarthritis: estimates from the global burden of disease 2010 study. Ann Rheum Dis 2014;73(07):1323-1330

2 Bijlsma JW, Berenbaum F, Lafeber FP. Osteoarthritis: an update with relevance for clinical practice. Lancet 2011;377(9783): 2115-2126

3 Lawrence RC, Felson DT, Helmick CG, et al; National Arthritis Data Workgroup. Estimates of the prevalence of arthritis and other rheumatic conditions in the United States. Part II. Arthritis Rheum 2008;58(01):26-35

4 Australian Orthopaedic Association National Joint Replacement Registry. Hip and knee arthroplasty: annual report. 2010

5 Bayliss LE, Culliford D, Monk AP, et al. The effect of patient age at intervention on risk of implant revision after total replacement of the hip or knee: a population-based cohort study. Lancet 2017; 389(10077):1424-1430

6 Price AJ, Longino D, Rees J, et al. Are pain and function better measures of outcome than revision rates after TKR in the younger patient? Knee 2010;17(03):196-199 
7 Skou ST, Roos EM, Laursen MB, et al. A randomized, controlled trial of total knee replacement. N Engl J Med 2015;373(17):1597-1606

8 Bernstein M, Reidler J, Fragomen A, Rozbruch SR. Ankle distraction arthroplasty: indications, technique, and outcomes. J Am Acad Orthop Surg 2017;25(02):89-99

9 Sandvall BK, Cameron TE, Netscher DT, Epstein MJ, Staines KG, Petersen NJ. Basal joint osteoarthritis of the thumb: ligament reconstruction and tendon interposition versus hematoma distraction arthroplasty. J Hand Surg Am 2010;35(12):1968-1975

10 Lafeber FP, Intema F, Van Roermund PM, Marijnissen AC. Unloading joints to treat osteoarthritis, including joint distraction. Curr Opin Rheumatol 2006;18(05):519-525

11 van der Woude JAD, Wiegant K, van Roermund PM, et al. Five-year follow-up of knee joint distraction: clinical benefit and cartilaginous tissue repair in an open uncontrolled prospective study. Cartilage 2017;8(03):263-271

12 van der Woude JA, Wiegant K, van Heerwaarden RJ, et al. Knee joint distraction compared with total knee arthroplasty: a randomised controlled trial. Bone Joint J 2017;99-B(01):51-58

13 van der Woude JAD, Wiegant K, van Heerwaarden RJ, et al. Knee joint distraction compared with high tibial osteotomy: a randomized controlled trial. Knee Surg Sports Traumatol Arthrosc 2017;25(03):876-886

14 Wiegant K, van Roermund PM, Intema F, et al. Sustained clinical and structural benefit after joint distraction in the treatment of severe knee osteoarthritis. Osteoarthritis Cartilage 2013;21(11): 1660-1667

15 Intema F, Van Roermund PM, Marijnissen AC, et al. Tissue structure modification in knee osteoarthritis by use of joint distraction: an open 1-year pilot study. Ann Rheum Dis 2011;70(08): 1441-1446

16 Deie M, Ochi M, Adachi N, Kajiwara R, Kanaya A. A new articulated distraction arthroplasty device for treatment of the osteoarthritic knee joint: a preliminary report. Arthroscopy 2007;23(08):833-838

17 Aly TA, Hafez K, Amin O. Arthrodiastasis for management of knee osteoarthritis. Orthopedics 2011;34(08):e338-e343

18 Wiegant K, Van Roermund PM, van Heerwaarden RJ, et al. Total knee prosthesis after knee joint distraction treatment. J Surg Surgical Res 2015;1:66-71

19 van Valburg AA, van Roermund PM, Marijnissen AC, et al. Joint distraction in treatment of osteoarthritis (II): effects on cartilage in a canine model. Osteoarthritis Cartilage 2000;8(01):1-8
20 Karadam B, Karatosun V, Murat N, Ozkal S, Gunal I. No beneficial effects of joint distraction on early microscopical changes in osteoarthrotic knees. A study in rabbits. Acta Orthop 2005;76(01):95-98

21 Kajiwara R, Ishida O, Kawasaki K, Adachi N, Yasunaga Y, Ochi M. Effective repair of a fresh osteochondral defect in the rabbit knee joint by articulated joint distraction following subchondral drilling. J Orthop Res 2005;23(04):909-915

22 Yanai T, Ishii T, Chang F, Ochiai N. Repair of large full-thickness articular cartilage defects in the rabbit: the effects of joint distraction and autologous bone-marrow-derived mesenchymal cell transplantation. J Bone Joint Surg Br 2005;87(05):721-729

23 Nishino T, Ishii T, Chang F, et al. Effect of gradual weight-bearing on regenerated articular cartilage after joint distraction and motion in a rabbit model. J Orthop Res 2010;28(05):600-606

24 Nishino T, Chang F, Ishii T, Yanai T, Mishima H, Ochiai N. Joint distraction and movement for repair of articular cartilage in a rabbit model with subsequent weight-bearing. J Bone Joint Surg Br 2010;92(07):1033-1040

25 Wiegant K, Intema F, van Roermund PM, et al. Evidence of cartilage repair by joint distraction in a canine model of osteoarthritis. Arthritis Rheumatol 2015;67(02):465-474

26 Chen Y, Sun Y, Pan X, Ho K, Li G. Joint distraction attenuates osteoarthritis by reducing secondary inflammation, cartilage degeneration and subchondral bone aberrant change. Osteoarthritis Cartilage 2015;23(10):1728-1735

27 Struik T, Jaspers JEN, Besselink NJ, et al. Technical feasibility of personalized articulating knee joint distraction for treatment of tibiofemoral osteoarthritis. Clin Biomech (Bristol, Avon) 2017; 49:40-47

28 Saltzman CL, Hillis SL, Stolley MP, Anderson DD, Amendola A. Motion versus fixed distraction of the joint in the treatment of ankle osteoarthritis: a prospective randomized controlled trial. J Bone Joint Surg Am 2012;94(11):961-970

29 van der Woude JA, van Heerwaarden RJ, Spruijt S, et al. Six weeks of continuous joint distraction appears sufficient for clinical benefit and cartilaginous tissue repair in the treatment of knee osteoarthritis. Knee 2016;23(05):785-791

30 van der Woude JA, Welsing PM, van Roermund PM, Custers RJ, Kuchuk NO, Lafeber FP. Prediction of cartilaginous tissue repair after knee joint distraction. Knee 2016;23(05):792-795

31 Jansen MP, Van der Weiden GS, Custers RJ, et al. Long-term benefit and survival of knee joint distraction as treatment of severe knee osteoarthritis. OARSI World Congress; 2018; Liverpool, UK

Appendix A MEDLINE (Ovid) Search Strategy

\begin{tabular}{|l|}
\hline 1. Knee joint/ \\
\hline 2. distraction.mp. OR arthrodiatasis.mp \\
\hline 3. 1 . AND 2. \\
\hline
\end{tabular}

